\title{
Noninvasive assessment of liver steatosis in children: the clinical value of controlled attenuation parameter
}

\author{
Giovanna Ferraioli ${ }^{1 *+} \mathbb{D}$, Valeria Calcaterra ${ }^{2 \dagger}$, Raffaella Lissandrin ${ }^{1}$, Marinella Guazzotti ${ }^{3}$, Laura Maiocchi ${ }^{1}$, \\ Carmine Tinelli ${ }^{4}$, Annalisa De Silvestri ${ }^{4}$, Corrado Regalbuto ${ }^{2}$, Gloria Pelizzo ${ }^{3}$, Daniela Larizza ${ }^{2}$ and Carlo Filice ${ }^{1}$
}

\begin{abstract}
Background: To assess the clinical validity of controlled attenuation parameter (CAP) in the diagnosis of hepatic steatosis in a series of overweight or obese children by using the imperfect gold standard methodology.

Methods: Consecutive children referred to our institution for auxological evaluation or obesity or minor elective surgery were prospectively enrolled. Anthropometric and biochemical parameters were recorded. Ultrasound (US) assessment of steatosis was carried out using ultrasound systems. CAP was obtained with the FibroScan 502 Touch device (Echosens, Paris, France). Pearson's or Spearman's rank correlation coefficient were used to test the association between two study variables. Optimal cutoff of CAP for detecting steatosis was $249 \mathrm{~dB} / \mathrm{m}$. The diagnostic performance of dichotomized CAP, US, body mass indexes (BMI), fatty liver index (FLI) and hepatic steatosis index (HSI) was analyzed using the imperfect gold standard methodology.
\end{abstract}

Results: Three hundred five pediatric patients were enrolled. The data of both US and CAP were available for 289 children. Steatosis was detected in 50/289 (17.3\%) children by US and in 77/289 (26.6\%) by CAP. A moderate to good correlation was detected between CAP and BMI $(r=0.53)$, FLI $(r=0.55)$ and HSI $(r=0.56)$. In obese children a moderate to good correlation between CAP and insulin levels $(r=0.54)$ and HOMA-IR $(r=0.54)$ was also found. Dichotomized CAP showed a performance of 0.70 (sensitivity, 0.72 [0.64-0.79]; specificity, 0.98 [0.97-0.98], which was better than that of US (performance, 0.37; sensitivity, 0.46 [0.42-0.50]; specificity, 0.91 [0.89-0.92]), BMI (performance, 0.22; sensitivity, 0.75 [0.73-0.77]; specificity, 0.57 [0.55-0.60]) and FLI or HSI.

Conclusions: For the evaluation of liver steatosis in children CAP performs better than US, which is the most widely used imaging technique for screening patients with a suspicion of liver steatosis. A cutoff value of CAP of $249 \mathrm{~dB} / \mathrm{m}$ rules in liver steatosis with a very high specificity.

Keywords: Liver steatosis, Pediatric series, NAFLD, Obesity, Controlled attenuation parameter, Ultrasound, Transient elastography

\section{Background}

Non-alcoholic fatty liver disease (NAFLD) is becoming a major health problem in children with an increasing incidence due to sedentary lifestyles and hyper-caloric diets that are the main factors of the obesity epidemics. Ten years ago, it was estimated that in Europe roughly

\footnotetext{
* Correspondence: giovanna.ferraioli@unipv.it

${ }^{\dagger}$ Equal contributors

${ }^{1}$ Ultrasound Unit, Department of Infectious Diseases, Fondazione IRCCS Policlinico San Matteo, University of Pavia, Viale Camillo Golgi 19, Pavia 27100, Italy

Full list of author information is available at the end of the article
}

one obese child out of three was affected by hepatic steatosis [1]. A recent review on the burden of liver disease in Europe has reported an even higher prevalence (36-44\%) [2]. A study conducted in the United States has reported that children with NAFLD have an increased risk of liverrelated mortality and a survival significantly shorter than that observed in the general population of the same age and sex [3]. Thus, the diagnosis and treatment of NAFLD at an early stage is of outmost importance for limiting the progression of the pathology. Liver biopsy is the reference standard for the assessment of liver steatosis, however the 
procedure is invasive, has several limitations including intra and inter-observer variability in specimen's readings, and it's unpractical for screening purpose or to follow-up patients.

Ultrasound (US) is the imaging modality most widely used for the noninvasive assessment of liver steatosis. A good diagnostic accuracy of US for the detection of moderate to severe steatosis in children has been reported, with an area under the receiver operating characteristic (AUROC) curve of 0.87 [4]. However, US has a low sensitivity for the detection of mild steatosis [5].

Controlled attenuation parameter (CAP) is a method for the non-invasive assessment of liver steatosis. It measures the attenuation of the ultrasound beam that traverses the liver tissue, which increases in fatty liver, and is obtained by analyzing the ultrasound signal acquired by the transient elastography device (Fibroscan 502 Touch, Echosens, Paris, France) [6]. It has been shown that CAP has a good accuracy for quantifying liver steatosis also in pediatric patients [7].

The main aim of this study was to assess the clinical validity of CAP in the early diagnosis of hepatic steatosis in a series of overweight or obese children by using the imperfect gold standard methodology. Secondary aim was to analyze any association of CAP with liver stiffness values and with other indices of NAFLD.

\section{Methods}

\section{Design of the study and patients}

This was a single center cross-sectional study. From September 2012 to May 2016, consecutive children referred to our institution for auxological evaluation or obesity by their general practitioner or primary care pediatric consultant, scheduled for abdominal US examination and accepting to undergo also to CAP and liver stiffness measurements (LSM)s, were prospectively enrolled. Children referred to the outpatient clinic of the paediatric surgery department of our institution for minor elective surgery were also enrolled as controls.

The exclusion criteria were known secondary obesity conditions, the use of any medications, and concomitant chronic or acute illnesses.

According to the body mass indexes (BMI), the subjects were divided into three groups: obese subjects (group1): BMI that exceeded the 95th percentile for the age and sex; overweight subjects (group2): BMI 75th-95th percentile; normal weight subjects (group3): BMI $<75$ th percentile.

The study protocol was approved by the ethics committee of the Fondazione IRCCS Policlinico San Matteo (reference number 20120020673) and it was in accordance with the Helsinki Declaration of 1975, as revised in 2008. All the participants or their responsible guardians gave their written consent after being informed about the nature of the study.

\section{Physical examination and biochemical parameters}

The physical examination of the participants included evaluation of height, weight, body mass index (BMI), waist circumference, pubertal stage according to Marshall and Tanner, and measurement of the blood pressure.

Metabolic blood assays included fasting blood glucose, insulin, total cholesterol, high-density lipoprotein (HDL) cholesterol, triglycerides (TG), and transaminases. Insulin resistance was determined by the homeostasis model assessment for insulin resistance (HOMA-IR) using the formula: insulin resistance $=($ insulin $\times$ glucose $) / 22.5$.

Abnormalities in lipid fasting levels were considered for TG values exceeding the 95th percentile and HDL cholesterol values below the fifth percentile for age and sex. Impaired insulin sensitivity was defined with HOMA-IR that exceeded the 97.5th percentile for age and sex.

Metabolic syndrome (MS) was diagnosed according to Weiss using the criteria modified from those of the National Cholesterol Education Program's Adult Treatment Panel III (NCEP-ATPIII) and the World Health Organization [8]. Patients were classified as having MS if they met three or more of the following criteria for age and sex: BMI $>95$ th percentile, TG levels $>95$ th percentile, HDL cholesterol level $<5$ th percentile, systolic and/or diastolic blood pressure $>95$ th percentile and fasting blood glucose $>100 \mathrm{mg} / \mathrm{dl}$ and/or impaired insulin sensitivity with HOMA-IR $>97.5$ th percentile. In the definition by Weiss, BMI was chosen as a criterion for the MS because it correlates with visceral lipid depot, blood pressure and dyslipidemia. Although waist circumference is a good predictor of visceral adiposity in children, it might not detect differences in body proportions related to puberty and therefore no normative values exist for children and adolescents. Impaired insulin sensitivity was included because impaired fasting glucose is rare in childhood. Finally, blood pressure and fasting lipid levels were compared with population norms adjusted for age and sex.

Fatty liver index (FLI) and hepatic steatosis index (HSI) were obtained using the established formulas $[9,10]$. FLI is an algorithm based on BMI, waist circumference, triglycerides and gamma-glutamyl-transferase and has been developed to detect steatosis [9], whereas HSI includes alanine aminotransferase (ALT)/aspartate aminotransferase (AST) ratio, BMI, diabetes mellitus and sex, and has been developed to detect NAFLD [10].

\section{Ultrasound examination, controlled attenuation parameter and liver stiffness measurements}

The US assessment of liver steatosis was performed using the iU22 system (Philips Medical Systems, Bothell, USA) or the HI VISION Ascendus system (Hitachi Ltd, Japan) equipped with convex multifrequency probes. The evaluation of liver steatosis was based on a series of 
US findings including liver echogenicity, hepatorenal echo contrast, visualization of intrahepatic vessels, and visualization of liver parenchyma and the diaphragm. Steatosis was scored as follows: absent (score 0) steatosis was defined as normal liver echotexture; mild (score 1) steatosis as slight and diffuse increase in fine parenchymal echoes with normal visualization of diaphragm and portal vein borders; moderate (score 2) steatosis as moderate and diffuse increase in fine echoes with slightly impaired visualization of portal vein borders and diaphragm; severe (score 3) steatosis as fine echoes with poor or no visualization of portal vein borders, diaphragm, and posterior portion of the right lobe $[4,11]$. The operators performing the examinations had at least 5 years of experience in US studies.

CAP was obtained by using the FibroScan 502 Touch device with the $3.5 \mathrm{MHz} \mathrm{M}$ probe or $2.5 \mathrm{MHz} \mathrm{XL}$ probes. CAP is a method for noninvasively quantifying the fat in the liver. The device estimates liver stiffness in kiloPascal $(\mathrm{kPa})$ and liver steatosis in decibel/meter $(\mathrm{dB} /$ $\mathrm{m})$. The principles of CAP have been described elsewhere [6]. CAP estimates the attenuation of the ultrasound waves at the central frequency of the probe of the Fibroscan device, and is guided by vibration-controlled transient elastography, ensuring that the operator automatically obtains the attenuation value of the liver. CAP was computed only when the associated LSM was valid and using the same signals as the one used to measure liver stiffness. As reported in the literature, only LSMs with 10 validated measurements and an interquartile range/median (IQR/M) $<30 \%$ for values higher than 7.1 $\mathrm{kPa}$ were considered reliable [12]. There are no recommendations for successful CAP measurement. Examinations with no successful measurements after 10 attempts were deemed failures. Following a fast of at least $6 \mathrm{~h}$, measurements were performed with the $M$ probe when the skin to liver capsule distance, estimated with US, was $\leq 25 \mathrm{~mm}$, otherwise the XL probe was used. In subjects with a thoracic perimeter less than $75 \mathrm{~cm}$ CAP measurements were not performed because the CAP is not available yet on the $5 \mathrm{MHz}$ S probe, which is specifically designed for the assessment of these subjects. In each patient, the LSMs and CAP measurements were performed by the same physician who had performed the US exam.

\section{Statistical analysis}

Power considerations: The comparison between children with obesity and children non obese, was used to investigate the discriminant validity of CAP. In the hypothesis of a significant percentage of steatosis of 50 in the first group and 20 in the second [4], a sample size of 80 in each group will have more than $90 \%$ power to detect that difference using a the two-sided $\mathrm{Z}$ test with pooled variance with a 0.01 significance level. In view of the possibility of missing data, at least 100 patients and 100 controls should be enrolled.

Descriptive statistics were produced for demographic characteristics for this study sample of patients. The Shapiro-Wilk test was used to test the normal distribution of quantitative variables. When quantitative variables were normally distributed, the results were expressed as the mean value and standard deviation (SD), otherwise median and interquartile range (IQR; 25th-75th percentile) were reported. Qualitative variables were summarized as counts and percentages. Pearson's correlation coefficient was used to test the association between two quantitative continuous variables; while Spearman's rank correlation coefficient was used to test the association between two ranked variables, or one ranked variable and one quantitative continuous variable. The correlations were categorized as follows: 0.00 to 0.25 none or slight; 0.25 to 0.50 fair to moderate; 0.50 to 0.75 moderate to good; 0.75 to 1.00 almost perfect [13].

The results of blood tests were missed for about $20 \%$ of the subjects; thus in order to calculate FLI and HSI we performed a multiple imputation of missing data. Ten datasets were created fitting regression models with TG, GGT, AST, ALT, glycaemia, waist circumference as dependent and $\mathrm{BMI}$, gender and age independent variables.

The diagnostic performance of CAP, BMI with AST and/ or ALT abnormal, HSI and FLI on diagnosing liver steatosis compared to the US score (gold standard) was assessed using receiver operating characteristic (ROC) curves and the area under the ROC (AUROC) curve analysis.

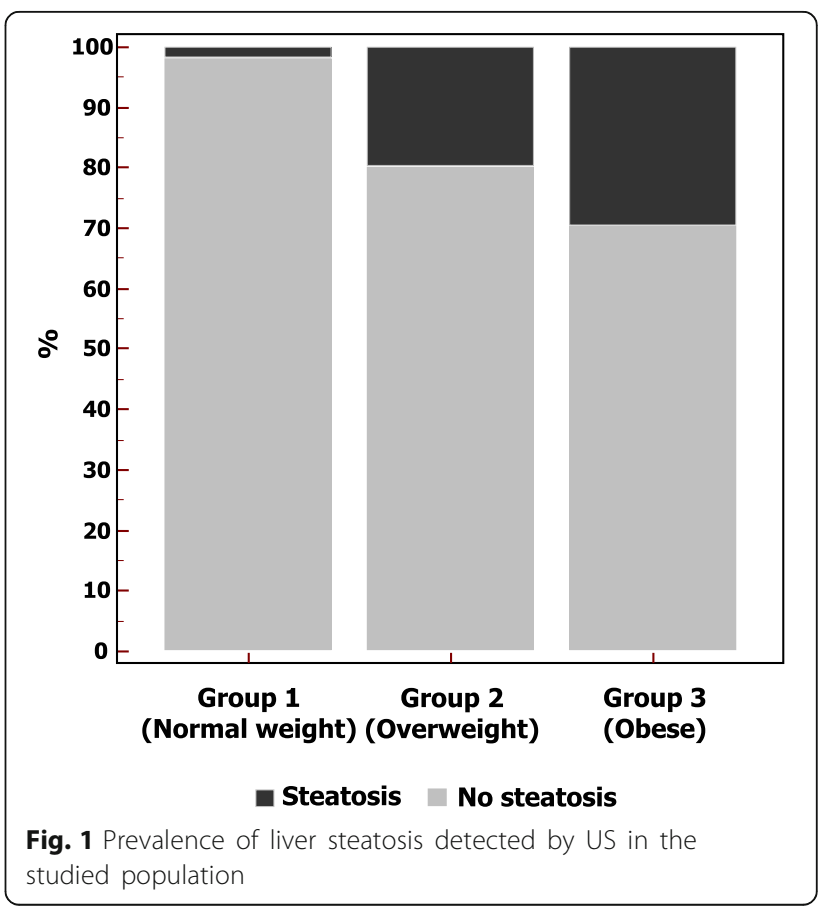


As a subject's true disease status is seldom known with certainty, errors made by the gold standard mean that the sensitivity and specificity calculated for the new test are biased, and do not correctly estimate the new method's sensitivity and specificity. Therefore we fitted two-level Bayesian latent class models with discrete latent variables to estimate sensitivity, specificity, positive predictive value (PPV), and negative predictive value (NPV) of diagnostic tests (imperfect gold standard methodology), as previously reported [14]. Briefly, the observed results of the diagnostic tests are considered as a measure, prone to error, of an unobservable (latent) dichotomous variable, i.e. the true disease status of each subject.

To fit the model, the CAP was categorized as $<249 \mathrm{~dB} / \mathrm{m}$ and $>=249 \mathrm{~dB} / \mathrm{m}$, i.e. the optimal cutoff obtained from our data (dichotomized CAP-dCAP), HSI and FLI as $<0.3$ and $>=0.3$, i.e. the value to rule out NAFLD or steatosis, respectively $[9,10]$. To improve the performance of the models the BMI of patients (categorized according to age as normal vs overweight/obese and with AST and/or ALT abnormal) was added as fourth test, since it has been previously used as an indicator of steatosis $[15,16]$.

$P<0.05$ was considered statistically significant. All tests were two-sided. The data analysis was performed with the STATA statistical package (release 14.0, 2015, Stata Corporation, College (Station, Texas, USA)

\section{Results}

Overall 305 pediatric patients were enrolled of whom 199 (103 males, 96 females; mean age, 11.5 years ([SD: 2.7]; range, 4.1-17.4) were referred for auxological evaluation or obesity and 106 (64 males, 42 females; mean age, 9.9 [SD: 3.3 years] for minor elective surgery. 100/199 (50.3\%) children referred for auxological evaluation or obesity were overweight (54 males, 46 females; mean age, 11.7 [SD: 2.6] years) and 99/199 (49.7\%) children were obese (50 males, 49 females; mean age, 11.0 [SD: 2.9] years). The prevalence of liver steatosis estimated using US was 24.1\%. 14/106 (13.2\%) children referred for minor elective surgery were overweight ( 7 males, 7 females; mean age, 9.2 [SD: 2.5] years) and 2/106 (1.9\%) children were obese

Table 1 Demographic, clinical and biochemical data of the studied population

\begin{tabular}{|c|c|c|c|c|c|}
\hline Variabile & $\begin{array}{l}\text { Overall } \\
N=305\end{array}$ & $\begin{array}{l}\text { Group1 } \\
\text { (Normal) } \\
N=106\end{array}$ & $\begin{array}{l}\text { Group2 } \\
\text { (Overweight) } \\
N=100\end{array}$ & $\begin{array}{l}\text { Group3 } \\
\text { (Obese) } \\
N=99\end{array}$ & $P$ value \# \\
\hline Gender, female, N (\%) & $135(44.8)$ & $40(38.5)$ & $46(46.9)$ & $49(49.4)$ & 0.25 \\
\hline Age & $10.9(3.0)$ & $10.1(3.3)$ & $11.8(2.6)$ & $11.0(2.95)$ & $<0.001^{2}$ \\
\hline Weight, kilograms & $51.2(19.9)$ & $35.2(12.2)$ & $54.8(13.8)$ & $63.9(20.4)$ & $<0.001^{*}$ \\
\hline Height, meters & $1.46(0.16)$ & $1.41(0.17)$ & $1.51(0.14)$ & $1.48(0.16)$ & $0.001^{1,3}$ \\
\hline $\mathrm{BMl}, \mathrm{kg} / \mathrm{m}^{2}$ & $23.1(5.7)$ & $17.2(2.8)$ & $23.6(2.3)$ & $28.4(4.4)$ & $<0.001^{*}$ \\
\hline Waist circumference, cm & $76.7(17.2)$ & $55.3(11.5)$ & $79.82(9.1)$ & $88.48(11.8)$ & $<0.001^{*}$ \\
\hline Systolic blood pressure, $\mathrm{mmHg}$ & $107.8(10.4)$ & $110.9(8.54)$ & $106.3(10.03)$ & $106.9(11.5)$ & $0.02^{1}$ \\
\hline Diastolic blood pressure, $\mathrm{mmHg}$ & $68.7(8.3)$ & $70.35(7.63)$ & $68.15(7.83)$ & $68.2(9)$ & 0.22 \\
\hline Total cholesterol, mg/dl & $160.3(29.5)$ & $156.4(29.4)$ & $160.6(28.3)$ & $162.6(30.5)$ & 0.44 \\
\hline HDL cholesterol, mg/dl & $48.9(10.8)$ & $52.7(12.3)$ & $49.4(10.6)$ & $46.2(9.4)$ & $0.001^{1}$ \\
\hline AST, IU/L & $22.6(8.69)$ & $22.7(6.45)$ & $21.8(9.01)$ & $23.2(9.9)$ & 0.56 \\
\hline$A L T, I U / L$ & $20.8(17.2)$ & $15.1(5.4)$ & $21.4(19.6)$ & $25.0(19.8)$ & $0.001^{3}$ \\
\hline GGT, IU/L & $16.5(8.5)$ & $13.3(5.5)$ & $16.7(6.2)$ & $18.9(11.0)$ & $<0.001^{1,3}$ \\
\hline Fasting blood glucose, mg/dl & $76.6(10.4)$ & $76.0(11.9)$ & $76.7(10.1)$ & $77.1(9.4)$ & 0.78 \\
\hline Fasting insulin, microlU/ml & $12.7(9.1)$ & $9.07(6.1)$ & $12.69(8.5)$ & $13.2(10.0)$ & 0.34 \\
\hline HOMA-IR, \% & $2.47(1.9)$ & $1.83(1.22)$ & $2.41(1.71)$ & $2.6(2.17)$ & 0.41 \\
\hline Triglycerides, mg/dl & $72.2(35.1)$ & $63.6(29.2)$ & $75.3(40.2)$ & $74.8(32.9)$ & 0.09 \\
\hline Fatty liver index & $0.18(0.21)$ & $0.03(0.08)$ & $0.14(0.12)$ & $0.3(0.2)$ & $<0.001^{*}$ \\
\hline Hepatic steatosis index & $0.46(0.34)$ & $0.13(0.18)$ & $0.45(0.26)$ & $0.7(0.2)$ & $<0.001^{*}$ \\
\hline CAP, decibel/meter & $228.5(50.34)$ & $200.5(39.36)$ & $231.6(51.46)$ & $254.6(44.4)$ & $<0.001^{*}$ \\
\hline LSM, kiloPascal & $4.70(.93)$ & $4.59(.96)$ & $4.73(.92)$ & $4.80(.9)$ & 0.30 \\
\hline Metabolic syndrome, N (\%) & $12(6.09)$ & $0(0)$ & $7(9.2)$ & $5(6.2)$ & 0.143 \\
\hline
\end{tabular}

BMI body mass index; HDL high-density lipoprotein; AST aspartate aminotransferase; $A L T$ alanine aminotransferase; GGT gamma glutamyltransferase; HOMA-IR homeostasis model assessment for insulin resistance; CAP controlled attenuation parameter; LSM liver stiffness measurement. Values are reported as mean and standard deviation unless otherwise specified

\# $\mathrm{P}$ values refer to differences among three groups; ${ }^{1}$ normal vs. overweight, ${ }^{2}$ normal vs obese, ${ }^{3}$ overweight vs obese: $p<0.05 ;{ }^{*}$ for all comparisons: $p<0.01$ 
(2 females; mean age, 11.5 [SD: 6.5]). The prevalence of liver steatosis estimated using US was $2.8 \%$.

The children of our cohort were assigned to group1, group2, or group3 according to their BMI. The prevalence of hepatic steatosis detected by US in the three groups is shown in Fig. 1. The demographic, clinical and biochemical data of our series are reported in Table 1.

Overall, MS occurred in five (6.2\%) obese children, in seven (9.2\%) overweight children and none child with normal weight $(p=0.12)$; no statistically significant difference was found between boys $(9 / 99 ; 9.9 \%)$ and girls $(3 / 98,3.06 \% ; p=0.08)$.

One component of the MS was present in $46(56.8 \%)$ obese children, 51 (67.1\%) overweight children and 9 (22.5\%) children with normal weight. Two components of the MS were found in 30 (37.0\%) obese children, 18 (23.7\%) overweight children and one (2.5\%) child with normal weight.

Clinical and biochemical characteristics of the children with or without liver steatosis detected by US are reported in Table 2. Significant differences were found

Table 2 Clinical and biochemical characteristics of the children with and without liver steatosis detected with ultrasound

\begin{tabular}{|c|c|c|c|}
\hline \multirow[t]{2}{*}{ Variable } & \multicolumn{2}{|l|}{ Liver steatosis $^{a}$} & \multirow{2}{*}{$\begin{array}{l}P \\
\text { value }\end{array}$} \\
\hline & Yes $(N=50)$ & No $(N=239)$ & \\
\hline Age, years & $11.6(2.5)$ & $10.8(3.04)$ & 0.06 \\
\hline Weight, kg & $62.2(16.0)$ & $48.8(19.7)$ & $<0.001$ \\
\hline Height, cm & $1.54(1.4-1.6)$ & $1.47(1.3-1.6)$ & .023 \\
\hline $\mathrm{BMI}, \mathrm{kg} / \mathrm{m}^{2}$ & $26.8(4.1)$ & $22.2(5.5)$ & $<0.001$ \\
\hline Waist circumference, $\mathrm{cm}$ & $86.58(11.43)$ & $74.1(17.4)$ & $<0.001$ \\
\hline Systolic blood pressure, mmHg & $107.65(11.66)$ & $108.0(10.1)$ & 0.85 \\
\hline Diastolic blood pressure, $\mathrm{mmHg}$ & $69.38(7.12)$ & $68.8(8.5)$ & 0.67 \\
\hline Total cholesterol, mg/dl & $159.1(29.13)$ & $160.6(29.8)$ & 0.75 \\
\hline HDL cholesterol, mg/dl & $46.9(10.34)$ & $49.28(11.0)$ & 0.19 \\
\hline AST, IU/L & $24.6(10.2)$ & $22.18(8.3)$ & 0.10 \\
\hline$A L T, I U / L$ & $32.6(26.3)$ & $18.16(13.2)$ & $<0.001$ \\
\hline GGT, IU/L & $15.2(6.8)$ & $22.4(12.3)$ & $<0.001$ \\
\hline Fasting blood glucose, mg/dl & $76.2(9.1)$ & $76.8(10.6)$ & 0.72 \\
\hline Fasting insulin, microlU/ml & $15.7(10.5)$ & $11.8(8.4)$ & 0.012 \\
\hline HOMA-IR, \% & $3(2.2)$ & $2.3(1.8)$ & 0.04 \\
\hline Triglycerides, mg/dl & $86.0(49.4)$ & $69.1(30.6)$ & 0.004 \\
\hline Fatty liver index & $0.30(0.20)$ & $0.15(0.19)$ & $<0.001$ \\
\hline Hepatic steatosis index & $0.76(0.24)$ & $0.39(0.32)$ & $<0.001$ \\
\hline CAP, decibel/meter & $280.9(54.1)$ & $217.4(41.8)$ & $<0.001$ \\
\hline LSM, kiloPascal & $5.02(1.03)$ & $4.65(0.89)$ & 0.01 \\
\hline
\end{tabular}

$B M I$ body mass index; $H D L$ high-density lipoprotein; $A S T$ aspartate aminotransferase; $A L T$ alanine aminotransferase; GGT gamma glutamyltransferase; HOMA-IR homeostasis model assessment for insulin resistance; CAP: controlled attenuation parameter; LSM liver stiffness measurement

${ }^{\text {a }}$ Data [mean (SD)] of the 289 children for whom both US and CAP were available for the anthropometric measures, ALT $(p<0.001)$, fasting insulin $(p=0.01)$, HOMA-IR $(p=0.04)$, triglycerides $(p=0.004)$, FLI $(p<0.001)$, HSI $(p<0.001)$, CAP $(p<0.01)$, and LSM $(p=0.009)$.

\section{Controlled attenuation parameter}

LSMs were obtained in 299/305 (98.0\%) children. The six failures were due to narrow intercostal spaces. All LSMs were reliable. The M probe was used in $285 / 299$ (95.3\%) children, the XL probe in 10/299 (3.4\%) children and the $\mathrm{S}$ probe in $4 / 299$ (1.4\%) children. In $4 / 299$ $(1.4 \%)$ the XL probe was used before the availability of CAP. Since CAP is not available yet on the $\mathrm{S}$ probe, only the data of 291 children examined with the $M$ probe and XL probe were analyzed for the noninvasive assessment of liver steatosis with CAP. In two children US was not performed for technical reasons. Thus, the data of both CAP and US were available for 289 children. The mean LSM was 4.71 (SD: 0.93; range 2-7.8) $\mathrm{kPa}$.

The distribution of CAP values in the three groups of children and in children without and with liver steatosis detected by US are reported in Figs. 2 and 3. CAP values were significantly lower in children with normal weight compared to overweight and obese children $(p<0.001)$. No differences were observed in LSM between the three groups (4.59 kPa [SD: 0.96], $4.73 \mathrm{kPa}$ [SD: 0.92] and 4.79 $\mathrm{kPa}$ [SD: 0.90]; $p=0.30$ ).

Steatosis was detected by US in 50/289 (17.3\%) children and by CAP in $77 / 289$ (26.6\%).

A moderate to good correlation was detected between CAP and BMI $(r=0.53)$, FLI $(r=0.55)$ and HSI $(r=0.56)$. In the obese group a moderate to good correlation between CAP and insulin levels $(r=0.54)$ and HOMA-IR $(r=0.54)$ was also found. No significant correlation was found between CAP and the other study variables.

\section{Diagnostic accuracy of noninvasive parameters of liver steatosis using US as the reference standard}

The AUROCs of CAP, FLI, HSI, BMI + AST and/or ALT in the assessment of liver steatosis are reported in Fig. 4. The AUROC of CAP was 0.84 (95\% CI:0.78-0.89, $p<0.001$ ), which was higher than that of HSI (0.82; 95\% CI:0.75-0.87), FLI (0.76; 95\% CI:0.69-0.82), and BMI + AST and/or ALT (0.68; 95\% CI:0.64-0.77).

Comparisons of AUROCs showed that CAP was significantly superior to BMI + AST and/or ALT $(p<0.001)$ whereas the differences between CAP and HSI, CAP and FLI were not statistically significant $(p=0.58$ and $p=0.10$, respectively).

Because several data for FLI and HSI calculation were missed, we compared FLI and HSI sensitivity/ specificity calculated on complete cases and after 


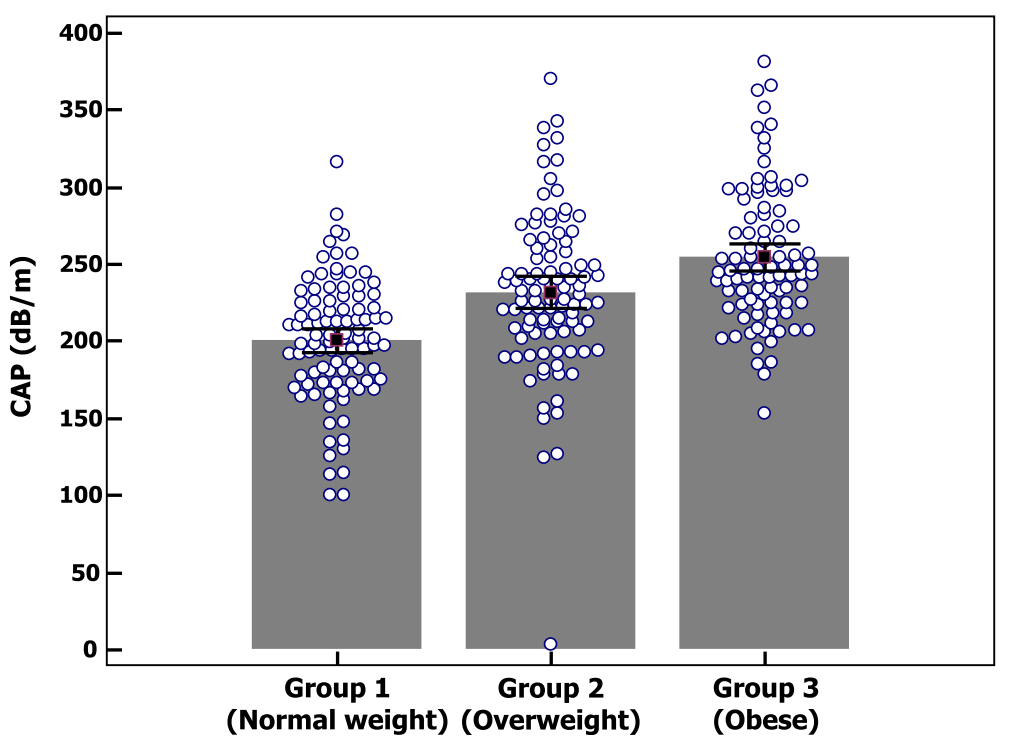

Fig. 2 Distribution of controlled attenuation parameter (CAP) values in the three groups of children

multiple imputation of missing data. On complete cases, FLI sensitivity and specificity were 0.44 (95\% CI: $0.30-0.59$ ) and 0.83 (95\% CI: 0.78-0.87), whereas HSI sensitivity and specificity were 0.93 (95\% CI: 0.84-0.99) and 0.48 (95\% CI: 0.42-0.54). After multiple imputation of missing data FLI and HSI sensitivity/specificity were similar to those calculated on complete cases (FLI sensitivity 0.41 [95\% CI: 0.27-0.55], specificity 0.84 (95\% CI: 0.79-0.89); HSI sensitivity 0.88 (95\% CI: $0.79-0.98)$, specificity 0.51 (95\% CI: 0.44-0.57).
Performance of the noninvasive parameters of liver steatosis using the methodology without the gold standard and the Bayesian latent class model analysis Because the models for the assessment of the performance without a gold standard require absolute independency of the diagnostic tests, we used two scenarios, one including dCAP, US, FLI, and BMI + AST and/or ALT, and the other one including the same parameters but with HSI instead of FLI. The results are reported in Table 3. In both scenarios dCAP showed the best performance with a sensitivity of 0.72 and a specificity of $0.98-1.00$.

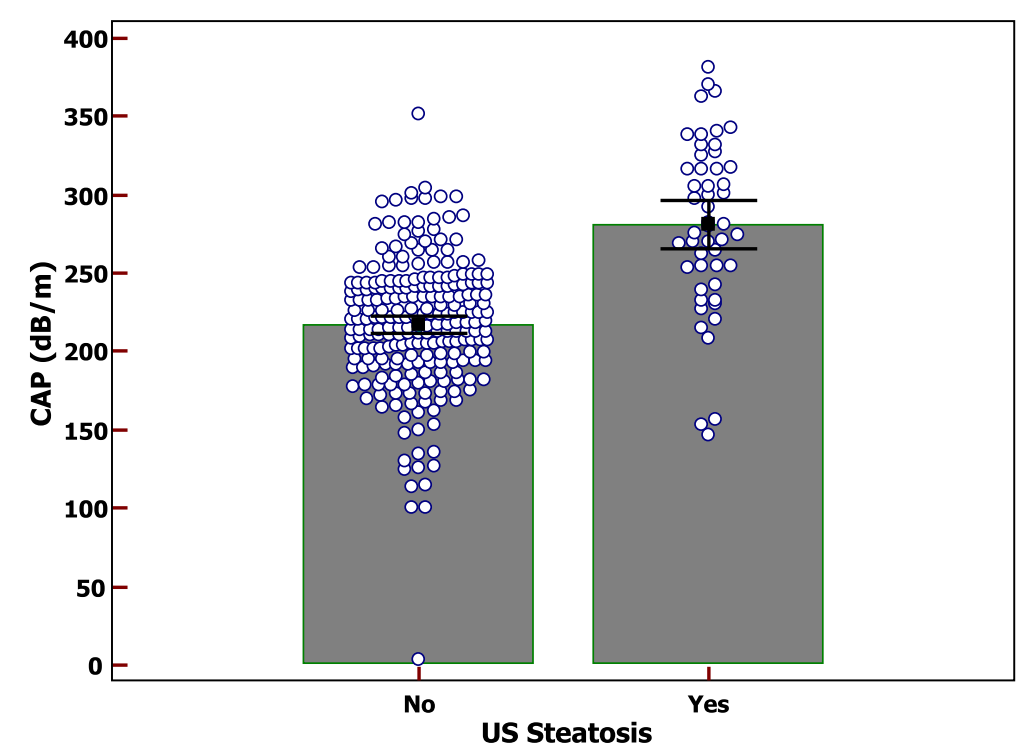

Fig. 3 Distribution of controlled attenuation parameter (CAP) values in children without and with liver steatosis detected by ultrasound 


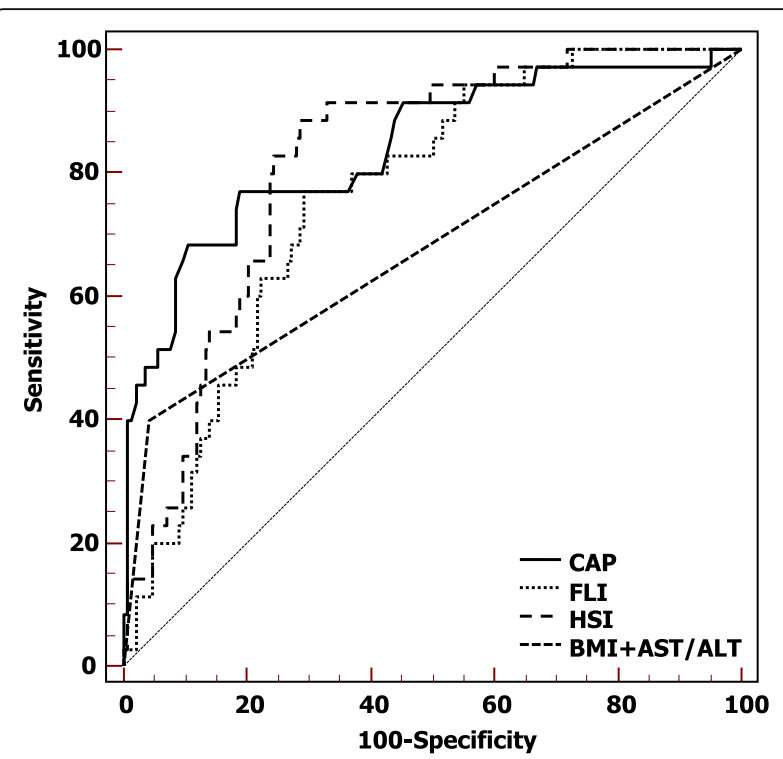

Fig. 4 AUROCs of controlled attenuation parameter (CAP), Fatty Liver Index (FLI), Hepatic Steatosis Index (HSI), Body Mass Index (BMI) + AST and/or ALT obtained using ultrasound as the reference standard

\section{Discussion}

The results of our study show that the diagnostic performance of CAP for the evaluation of liver steatosis was higher than that of US. Due to its noninvasiveness, availability, relatively low cost and repeatability because there is no exposition to ionizing radiation, US is the most widely used technique for screening patients with a suspicion of liver steatosis. Over the years, the technological advancement of the US systems has led to an increase of the sensitivity of technique, which has been reported to be 0.90 in detecting steatosis involving at least $20 \%$ of the hepatocytes [17]. However, for lower levels of fat infiltration a negative US result does not rule out the presence of liver steatosis. Especially in children, it is of outmost importance to diagnose NAFLD at an early stage because a modification of the diet and of the lifestyle can avoid the progression to end-stage liver disease, whose incidence seems higher than in adults $[18,19]$. Magnetic resonance spectroscopy is the most accurate method for the detection of fat in the liver, however the technique is costly and available only in few referral centers, and often requires sedation in children. CAP is a new noninvasive method that has shown promise in the assessment of steatosis also in pediatric patients. Recently, in a study performed in a small series of children in whom liver biopsy was carried out for clinical indications, a cut point of $225 \mathrm{~dB} / \mathrm{m}$ for predicting steatosis has been identified, with 0.87 sensitivity, 0.83 specificity, and AUROC 0.93 [7]. The results of our study show that a CAP cutoff value of $249 \mathrm{~dB} / \mathrm{m}$ rules in liver steatosis with a very high specificity. Moreover, steatosis was detected with CAP in 27 (9.3\%) children with normal US findings. This finding suggests that CAP could be a useful tool for the diagnosis of liver steatosis at an early stage.

Table 3 Diagnostic accuracy of the noninvasive parameters of liver steatosis in the studied population using the imperfect gold standard methodology

\begin{tabular}{|c|c|c|c|c|c|}
\hline Parameter & $\begin{array}{l}\text { Sensitivity } \\
(95 \% \mathrm{Cl})\end{array}$ & $\begin{array}{l}\text { Specificity } \\
(95 \% \text { Cl) }\end{array}$ & $\begin{array}{l}\text { PPV } \\
(95 \% \text { Cl) }\end{array}$ & $\begin{array}{l}\text { NPV } \\
(95 \% \text { Cl) }\end{array}$ & Performance $^{\mathrm{a}}$ \\
\hline \multicolumn{6}{|c|}{ Analysis with the imperfect gold standard methodology: scenario 1} \\
\hline dCAP & $\begin{array}{l}0.72 \\
(0.64-0.79)\end{array}$ & $\begin{array}{l}0.98 \\
(0.97-0.98)\end{array}$ & $\begin{array}{l}0.93 \\
(0.90-0.96)\end{array}$ & $\begin{array}{l}0.89 \\
(0.86-0.92)\end{array}$ & 0.70 \\
\hline Ultrasound & $\begin{array}{l}0.46 \\
(0.42-0.50)\end{array}$ & $\begin{array}{l}0.91 \\
(0.89-0.92)\end{array}$ & $\begin{array}{l}0.68 \\
(0.62-0.73)\end{array}$ & $\begin{array}{l}0.80 \\
(0.78-0.81)\end{array}$ & 0.37 \\
\hline Fatty Liver index & $\begin{array}{l}0.73 \\
(0.72-0.74)\end{array}$ & $\begin{array}{l}0.27 \\
(0.25-0.28)\end{array}$ & $\begin{array}{l}0.30 \\
(0.29-0.31)\end{array}$ & $\begin{array}{l}0.7 \\
(0.69-0.7)\end{array}$ & 0 \\
\hline $\mathrm{BMI}+\mathrm{AST}$ and/or ALT & $\begin{array}{l}0.75 \\
(0.73-0.77)\end{array}$ & $\begin{array}{l}0.57 \\
(0.55-0.60)\end{array}$ & $\begin{array}{l}0.43 \\
(0.41-0.45)\end{array}$ & $\begin{array}{l}0.84 \\
(0.82-0.86)\end{array}$ & 0.22 \\
\hline \multicolumn{6}{|c|}{ Analysis with the imperfect gold standard methodology: scenario 2} \\
\hline dCAP & $\begin{array}{l}0.72 \\
(0.69-0.78)\end{array}$ & $\begin{array}{l}1.00 \\
(1.00-1.00)\end{array}$ & $0.99(0.99-1)$ & $\begin{array}{l}0.89 \\
(0.88-0.91)\end{array}$ & 0.72 \\
\hline Ultrasound & $\begin{array}{l}0.43 \\
(0.38-0.50)\end{array}$ & $\begin{array}{l}0.99 \\
(0.99-1.00)\end{array}$ & $\begin{array}{l}0.96 \\
(0.94-0.98)\end{array}$ & $\begin{array}{l}0.8 \\
(0.78-0.82)\end{array}$ & 0.42 \\
\hline Hepatic Steatosis Index & $\begin{array}{l}0.85 \\
(0.84-0.85)\end{array}$ & $\begin{array}{l}0.33 \\
(0.32-0.35)\end{array}$ & $\begin{array}{l}0.35 \\
(0.34-0.36)\end{array}$ & $\begin{array}{l}0.83 \\
(0.82-0.84)\end{array}$ & 0.18 \\
\hline $\mathrm{BMI}+\mathrm{AST}$ and/or ALT & $\begin{array}{l}0.73 \\
(0.69-0.77)\end{array}$ & $\begin{array}{l}0.73 \\
(0.69-0.77)\end{array}$ & $\begin{array}{l}0.54 \\
(0.49-0.58)\end{array}$ & $\begin{array}{l}0.86 \\
(0.84-0.88)\end{array}$ & 0.54 \\
\hline
\end{tabular}

PPV positive predictive value; NPV negative predictive value; CI 95\% Credible Interval; $d C A P$ dichotomized controlled attenuation parameter; $B M I$ body mass index; AST aspartate aminotransferase; $A L T$ alanine aminotransferase

a Performance $=$ Sensitivity + Specificity -100 
In our series, children with liver steatosis showed LSMs significantly higher than those observed in children without steatosis by some $0.5 \mathrm{kPa}$. Although this difference was not relevant from a clinical point of view because these values were still in the normal range, we would like to underline that significant liver fibrosis was observed in one child with liver steatosis and in none child without. This finding confirms that liver steatosis is not always a benign condition, thus care should be taken to assess the disease at an early stage. On this regard, CAP seems a very useful tool.

FLI and HSI are biochemical indices of hepatic steatosis that have been derived and a currently used in the adult population, whereas data on children are scarce. In our series, similar to what observed in adults, a moderate to good correlation of CAP with these indices was detected, indicating that FLI and HSI could be used as noninvasive biomarkers of liver steatosis in the pediatric population as well. However, it has been reported that these indices have insufficient diagnostic accuracy for diagnosing or excluding NAFLD in severely obese children [20].

This study has limitations. First, we used the methodology without a gold standard since liver biopsy is not indicated for screening purpose even in a high risk population. The latent class analysis method for estimating test accuracy in the absence of a gold standard is well documented and has been applied in several studies [21]. Second, the biochemical data were not available for all children, thus we used the multiple imputation of missing data. However, the sensitivity and specificity were similar to those calculated with the complete data. Third, the children in our study were enrolled from pediatric patients referred to the hospital for auxological evaluation or obesity or elective minor surgery, therefore the results may not be applicable to the general population. Forth, since we used US as the reference standard to calculate the cutoff value of CAP for the detection of steatosis, we could have underestimated liver steatosis in our cohort. It is likely that with a lower cutoff, as the one obtained in the series of Desai et al [7] where liver biopsy was the reference standard, the performance of CAP would have been even higher than observed in our population.

\section{Conclusions}

For the evaluation of liver steatosis in children CAP performs better than US, which is the most widely used imaging technique for screening patients with a suspicion of liver steatosis. A cutoff value of CAP of $249 \mathrm{~dB} / \mathrm{m}$ rules in liver steatosis with a very high specificity.

\section{Abbreviations}

ALT: Alanine aminotransferase; AST: Aspartate aminotransferase; AUROC: Area under the receiver operating characteristic; BMI: Body mass index;

CAP: Controlled attenuation parameter; Cl: Confidence interval; dB/ m: Decibel/meter; dCAP: Dichotomized CAP; FLI: Fatty liver index; HDL: Highdensity lipoprotein; HOMA-IR: Homeostasis model assessment for insulin resistance; HSI: Hepatic steatosis index; IQR: Interquartile range; IQR/ M: Interquartile range/median; KPa: kiloPascal; LSM: Liver stiffness measurements; MS: Metabolic syndrome; NAFLD: Non-alcoholic fatty liver disease; NCEP-ATP: National Cholesterol Education Program's Adult Treatment Panel; NPV: Negative predictive value; PPV: Positive predictive value; ROC: Receiver operating characteristic; SD: Standard deviation; TG: Triglycerides; US: Ultrasound

\section{Acknowledgements}

The authors would like to thank Ms. Nadia Locatelli, Secretary of the Ultrasound Unit, for her valuable help in complying with the study protocol.

\section{Funding}

This work has been supported by a grant given by the Italian Ministry of Health [RF-2011-02347402]. Carlo Filice is the recipient of the grant. The funding body had no role in the design of the study and collection, analysis, and interpretation of data and in writing the manuscript.

\section{Availability of data and materials}

The datasets generated and/or analyzed during the current study are available from the corresponding author on request.

\section{Authors' contributions}

GF and CF developed the study protocol; VC, RL, MG, LM, CR performed the study and collected the data; CT and ADS analyzed and interpreted the patients' data; GF wrote the manuscript; VC, RL, MG, LM, CT, ADS, CR, GP, DL, CF critically revised the draft of the manuscript for important intellectual content; all authors read and approved the final manuscript and agreed to be accountable for all aspects of the work in ensuring that questions related to the accuracy or integrity of any part of the work are appropriately investigated and resolved.

\section{Competing interests}

GF has served as a speaker for Philips Healthcare, Hitachi Ltd., Toshiba Medical Systems. CF has served as a speaker for Philips Healthcare, Hitachi Ltd., Esaote S.p.A., and has received research funding from Esaote S.p.A., Bracco Imaging, Hitachi Ltd., Toshiba Medical Systems. VC, RL, MG, LM, CT, ADS, CR, GP, DL have no conflict of interest to declare.

\section{Consent for publication}

Not applicable.

\section{Ethics approval and consent to participate}

The study was approved by the ethics committee of the Fondazione IRCCS Policlinico San Matteo, Pavia, reference number 20120020673, and performed in accordance with the Declaration of Helsinki. All the participants or their responsible guardians gave their written consent after being informed about the nature of the study.

\section{Publisher's Note}

Springer Nature remains neutral with regard to jurisdictional claims in published maps and institutional affiliations.

\section{Author details}

'Ultrasound Unit, Department of Infectious Diseases, Fondazione IRCCS Policlinico San Matteo, University of Pavia, Viale Camillo Golgi 19, Pavia 27100, Italy. ${ }^{2}$ Pediatric Unit, Department of the Mother and Child Health, Fondazione IRCCS Policlinico San Matteo, University of Pavia, Pavia, Italy. ${ }^{3}$ Pediatric Surgery Unit, Department of the Mother and Child Health,

Fondazione IRCCS Policlinico San Matteo, University of Pavia, Pavia, Italy.

${ }^{4}$ Clinical Epidemiology and Biometric Unit, Fondazione IRCCS Policlinico San Matteo, Pavia, Italy.

Received: 27 January 2017 Accepted: 20 April 2017

Published online: 04 May 2017

References

1. Lobstein T, Jackson-Leach R. Estimated burden of paediatric obesity and co-morbidities in Europe. Part 2. Numbers of children with indicators of 
obesity-related disease. Int J Pediatr Obes. 2006;1(1):33-41. doi:10.1080/ 17477160600586689.

2. Blachier M, Leleu H, Peck-Radosavljevic M, Valla DC, Roudot-Thoraval F. The burden of liver disease in Europe: a review of available epidemiological data. J Hepatol. 2013;58(3):593-608. doi:10.1016/j.jhep.2012.12.005.

3. Feldstein $A E$, Charatcharoenwitthaya $P$, Treeprasertsuk S, Benson JT, Enders $F B$, Angulo $P$. The natural history of non-alcoholic fatty liver disease in children: a follow-up study for up to 20 years. Gut. 2009;58(11):1538-44. doi: 10.1136/gut.2008.171280

4. Shannon A, Alkhouri N, Carter-Kent C, Monti L, Devito R, Lopez R, et al. Ultrasonographic quantitative estimation of hepatic steatosis in children with NAFLD. J Pediatr Gastroenterol Nutr. 2011;53(2):190-5. doi:10.1097/ MPG.0b013e31821b4b61.

5. Lee SS, Park SH, Kim HJ, Kim SY, Kim MY, Kim DY, et al. Non-invasive assessment of hepatic steatosis: prospective comparison of the accuracy of imaging examinations. J Hepatol. 2010;52(4):579-85. doi:10.1016/j.jhep.2010. 01.008.

6. Sasso M, Beaugrand M, de Ledinghen V, Douvin C, Marcellin P, Poupon R, et al. Controlled attenuation parameter (CAP): a novel VCTE guided ultrasonic attenuation measurement for the evaluation of hepatic steatosis: preliminary study and validation in a cohort of patients with chronic liver disease from various causes. Ultrasound Med Biol. 2010;36(11):1825-35. doi: 10.1016/j.ultrasmedbio.2010.07.005.

7. Desai NK, Harney S, Raza R, Al-lbraheemi A, Shillingford N, Mitchell PD, et al. Comparison of controlled attenuation parameter and liver biopsy to assess hepatic steatosis in pediatric patients. J Pediatr. 2016;173:160-4. doi:10.1016/ j.jpeds.2016.03.021. e161.

8. Weiss R, Dziura J, Burgert TS, Tamborlane W, Taksali SE, Yeckel CW, et al. Obesity and the metabolic syndrome in children and adolescents. N Engl J Med. 2004;350(23):2362-74. doi:10.1056/NEJMoa031049.

9. Bedogni G, Bellentani S, Miglioli L, Masutti F, Passalacqua M, Castiglione A, et al. The Fatty Liver Index: a simple and accurate predictor of hepatic steatosis in the general population. BMC Gastroenterol. 2006;6:33. doi:10. 1186/1471-230X-6-33.

10. Lee JH, Kim D, Kim HJ, Lee CH, Yang Jl, Kim W, et al. Hepatic steatosis index: a simple screening tool reflecting nonalcoholic fatty liver disease. Dig Liver Dis. 2010;42(7):503-8. doi:10.1016/j.dld.2009.08.002.

11. Kim SH, Lee JM, Kim JH, Kim KG, Han JK, Lee KH, et al. Appropriateness of a donor liver with respect to macrosteatosis: application of artificial neural networks to US images-initial experience. Radiology. 2005;234(3):793-803. doi:10.1148/radiol.2343040142.

12. Boursier J, Zarski JP, de Ledinghen V, Rousselet MC, Sturm N, Lebail B, et al. Determination of reliability criteria for liver stiffness evaluation by transient elastography. Hepatology. 2013:57(3):1182-91. doi:10.1002/hep.25993.

13. Colton T, editor. Statistics in medicine. Boston: Little, Brown and Company; 1974

14. Ferraioli G, Tinelli C, De Silvestri A, Lissandrin R, Above E, Dellafiore C, et al. The clinical value of controlled attenuation parameter for the noninvasive assessment of liver steatosis. Liver Int. 2016;36(12):1860-6. doi:10.1111/liv.13207.

15. Peng CJ, Yuan D, Li B, Wei YG, Yan LN, Wen TF, et al. Body mass index evaluating donor hepatic steatosis in living donor liver transplantation. Transplant Proc. 2009;41(9):3556-9. doi:10.1016/j.transproceed.2009.06.235.

16. Rinella ME, Alonso E, Rao S, Whitington P, Fryer J, Abecassis M, et al. Body mass index as a predictor of hepatic steatosis in living liver donors. Liver Transpl. 2001;7(5):409-14. doi:10.1053/jtts.2001.23787.

17. Dasarathy S, Dasarathy J, Khiyami A, Joseph R, Lopez R, McCullough AJ. Validity of real time ultrasound in the diagnosis of hepatic steatosis: a prospective study. J Hepatol. 2009;51(6):1061-7. doi:10.1016/j.jhep.2009.09.001.

18. Berardis S, Sokal E. Pediatric non-alcoholic fatty liver disease: an increasing public health issue. Eur J Pediatr. 2014;173(2):131-9. doi:10. 1007/s00431-013-2157-6.

19. Rashid M, Roberts EA. Nonalcoholic steatohepatitis in children. J Pediatr Gastroenterol Nutr. 2000;30(1):48-53. doi:10.1097/00005176-200001000-00017.

20. Koot BG, van der Baan-Slootweg OH, Bohte $A E$, Nederveen AJ, van Werven $J R$, Tamminga-Smeulders $C L$, et al. Accuracy of prediction scores and novel biomarkers for predicting nonalcoholic fatty liver disease in obese children. Obesity (Silver Spring). 2013;21(3):583-90. doi:10.1002/oby.20173.

21. Rutjes AW, Reitsma JB, Coomarasamy A, Khan KS, Bossuyt PM. Evaluation of diagnostic tests when there is no gold standard. A review of methods. Health Technol Assess. 2007;11(50):iii. doi:10.3310/hta11500. ix-51.

\section{Submit your next manuscript to BioMed Central and we will help you at every step:}

- We accept pre-submission inquiries

- Our selector tool helps you to find the most relevant journal

- We provide round the clock customer support

- Convenient online submission

- Thorough peer review

- Inclusion in PubMed and all major indexing services

- Maximum visibility for your research

Submit your manuscript at www.biomedcentral.com/submit
C Biomed Central 